\title{
Special Issue: The 11th International Symposium on Electric and Magnetic Fields (EMF 2018)
}

On behalf of the Editorial Board, we are pleased to present a selection of papers related to the 11th International Symposium on Electric and Magnetic Fields (EMF 2018) held in Darmstadt, Germany, on 10-12 April, 2018.

The EMF Symposium series, whose first edition was held in Liège, Belgium, in 1992, aims at building a bridge between recent research advances in mathematical and numerical modelling of electromagnetic fields and the growing number of industrial problems requiring such techniques. The 11th edition was organized by the Institute for Accelerator Science and Electromagnetic Fields and the Centre for Computational Engineering of the Technische Universität Darmstadt, and attracted 95 participants from 19 countries.

Among the 84 presentations in the symposium program, 31 manuscripts were submitted for peer review. Fourteen papers were selected for publication in this special issue of the International Journal of Numerical Modelling: Electronic Networks, Devices and Fields. The high scientific and technical quality of the Symposium is well reflected in the quality of the manuscripts contained in this special issue.

Four broad topics were covered during the 11th edition of the symposium.

The first major topic considers the mathematical modelling of electromagnetic problems in view of their eventual numerical solution on computers, with contributions on the coupling of finite element and multipole methods, ${ }^{1}$ boundary element methods, ${ }^{2}$ inversion methods ${ }^{3}$ and electromagnetic transformation laws. ${ }^{4}$ The second major topic treats the numerical modelling of the electromagnetic behaviour of electric and magnetic materials, with contributions on the modelling of magnetoelectric composites ${ }^{5}$ and magnetic hysteresis. ${ }^{6,7}$ The third major topic concerns homogenization and reduced order models, with contributions to the time-domain homogenization of windings, ${ }^{8}$ extraction of capacitance matrices ${ }^{9}$ and lumped element modelling. ${ }^{10}$ The fourth major topic concerns application-specific numerical techniques, with contributions to the modelling of synchronous reluctance machines, ${ }^{11}$ graded surge arresters, ${ }^{12}$ high voltage power cables ${ }^{13}$ and DC-AC power converters. ${ }^{14}$

We express our gratitude to all the members of the EMF scientific committee-P. Alotto, J.P.A. Bastos, O. Biro, M. Clemens, S. Clenet, W.A. Cronje, A. Demenko, L. Dupré, J. Gyselinck, K. Hameyer, L. Kettunen, A. Kost, L. Krähenbühl, V. Mazauric, J. Melkebeek, G. Meunier, A. Nicolet, M. Petrun, A. Razek, M. Repetto, G. Rubinacci, R. Sabariego, J.K. Sykulski and M. Trlep-as well as to all the reviewers, who provided the necessary volunteer time and expertise to conduct a fair and detailed review, ensuring high publication standards for the selected manuscripts.

We also want to thank the staff from the Association des Ingénieurs de Montefiore (AIM), and especially C. Dizier, for their help organizing the EMF symposium series: the next edition will be held in Marseille, France, in July 2021.

Finally, we would also like to thank all the editorial staff of the International Journal of Numerical Modelling: Electronic Networks, Devices and Fields for their kind support during the whole process of preparation of this special issue.

We hope you will enjoy reading this selection of articles.

Herbert De Gersem ${ }^{1}$ Stefan Kurz ${ }^{2,3}$

Sebastian Schöps ${ }^{4}$

Christophe Geuzaine ${ }^{5}$

${ }^{1}$ Chair of Electromagnetic Field Theory, Technische Universität Darmstadt, Darmstadt, Germany

${ }^{2}$ Chair of Electromagnetism and Mathematical Modelling, Technische Universität Darmstadt, Darmstadt, Germany

${ }^{3}$ Bosch Center for Artificial Intelligence, Renningen, Germany

${ }^{4}$ Chair of Computational Electromagnetics, Technische Universität Darmstadt, Darmstadt, Germany

${ }^{5}$ Department of Electrical Engineering and Computer Science, Institut Montefiore B28, Université de Liège, Liège, Belgium 
Correspondence

Christophe Geuzaine, Department of Electrical Engineering and Computer Science, Institut Montefiore B28, Université de Liège, B-4000 Liège, Belgium. Email: cgeuzaine@uliege.be

\section{REFERENCES}

1. Casati D. Coupling finite elements and auxiliary sources for Maxwell's equations. Int J Numer Model. 2020;33:e2534.

2. Phan QA. 3D Eddy currents computation by BEM using the modified magnetic vector potential and the reduced magnetic scalar potential. Int J Numer Model. 2020;33:e2642.

3. Zhang G. Research on spatial reconstruction of arc plasma based on heuristic search and regularization inversion method. Int $J$ Numer Model. 2020;33:e2672.

4. Lehmann MC. Encoding electromagnetic transformation laws for dimensional reduction. Int J Numer Model. 2020;33:e2747.

5. Do TA. 3D FEM modeling and study of novel structure of magnetoelectric composites. Int J Numer Model. 2020;33:e2671.

6. Bavendiek G. Modeling of history-dependent magnetization in the finite element method on the example of a post-assembly rotor magnetizer. Int J Numer Model. 2020;33:e2674.

7. Elfgen S. Hysteresis and Eddy-current losses in electrical steel utilizing edge degradation due to cutting effects. Int J Numer Model. 2020; 33:e2781.

8. Niyomsatian K. Time-domain homogenization of multiturn windings based on RL Cauer ladder networks. Int J Numer Model. 2020;33: e2649.

9. Drandić A. Transformer capacitance matrix computation using 3D boundary element method and adaptive cross approximation. Int $J$ Numer Model. 2020;33:e2669.

10. Lannoo J. Improved air gap permeance model to characterise the transient behaviour of electrical machines using MEC method. Int $J$ Numer Model. 2020;33:e2749.

11. Neusüs S. Numerical evaluation of permanent magnet assisted synchronous reluctance rotor topologies using a simplified magnetostatic model. Int J Numer Model. 2020;33:e2574.

12. D'Angelo LAM. Electroquasistatic quasi-3D finite-element simulation of a graded surge arrester. Int J Numer Model. 2020;33:e2575.

13. Joergens C. Fast calculation of steady-state charge distribution in high voltage power cables. Int J Numer Model. 2020;33:e2713.

14. Pels A. Efficient simulation of DC-AC power converters using multirate partial differential equations. Int J Numer Model. 2020;33:e2683. 